\section{Linfoma de células grandes B originado en enfermedad de Castleman. Reporte de un caso y revisión de la literatura}

\author{
ALEJANDRO AVILÉS-SALAS ${ }^{1,3}$, MYRNA CANDELARIA $^{2}$, \\ DIANA BRISA SEVILLA-LIZCANO ${ }^{1}$, SEBASTIÁN BURGOS ${ }^{2}$

\section{Large B-cell lymphoma coexisting with Castleman's disease. Report of one case}

We report a 73-year-old female patient with Castleman's disease coexistent with large B cell type non-Hodgkin's lymphoma in a right axillary lymphadenopathy. An excisional biopsy was performed: microscopically, the lymph node revealed the presence of numerous plasma cells and small lymphoid cells characteristic of Castleman's disease. An analysis of another portion of the specimen revealed lymphoid cells with large abnormal nuclei gathered locally that were CDD 79+, CD 38+ and MUM-1+ as well as positive for Kaposi sarcoma-associated herpesvirus and negative for Epstein Barr virus encoded RNA-1 (EBER).

(Rev Med Chile 2017; 145: 945-949)

Key words: Giant Lymph Node Hyperplasia; Herpesvirus 8, Human; Lymph Nodes; Lymphoma Large B-Cell, Diffuse.

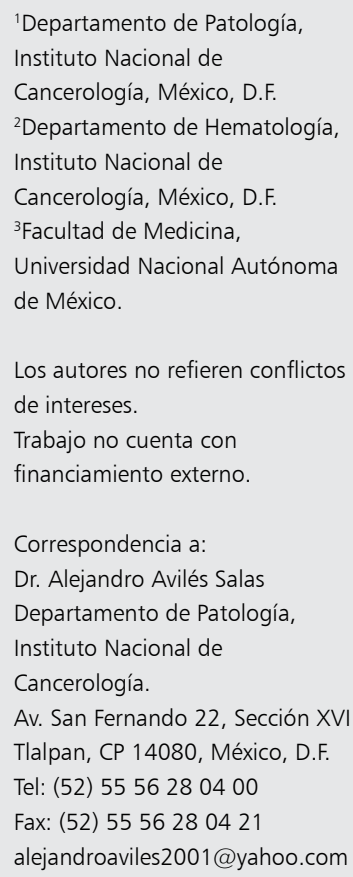

$\mathrm{E}$ 1 linfoma de células grandes B, originado en enfermedad de Castleman (LCGBEC), está compuesto por una proliferación monoclonal de células linfoides infectadas por el herpesvirus asociado a sarcoma de Kaposi/virus herpes humano tipo 8 (HVSK/VHH8) semejantes a plasmablastos con expresión de IgM, IL-6 viral y patrón de tinción nuclear para el antígeno nuclear de latencia-1 (LANA-1), en el contexto de enfermedad de Castleman (EC) habitualmente multicéntrica ${ }^{1,2}$. Usualmente se asocia a pacientes con infección por el virus de inmunodeficiencia humana (VIH); sin embargo, se han informado casos en pacientes inmunocompetentes ${ }^{3,4}$. Se utiliza el término plasmablástico debido a que las células morfológicamente recuerdan células plasmáticas; sin embargo, corresponden a una célula plasmática con producción de $\operatorname{IgM}$, sin hipermutación somática de inmunoglobulinas 5 . Informamos el caso de una mujer de 73 años de edad VIH- con EC coexistente con linfoma de células grandes B (LCGB) en un ganglio linfático axilar.

\section{Caso clínico}

Paciente de sexo femenino de 73 años de edad sin antecedentes mórbidos relevantes. Inició su enfermedad 6 años previos a su hospitalización con adenomegalia axilar derecha de crecimiento progresivo, acompañada de diaforesis, sin manejo terapéutico. Posteriormente, tres meses previos a su ingreso se palpó adenomegalia axilar derecha de aproximadamente $7 \mathrm{~cm}$. Acudió con médico oncólogo que realizó biopsia incisional del ganglio linfático con la sospecha diagnóstica de linfoma, motivo por el cual fue referida al Instituto Nacional de Cancerología para su valoración y manejo. A su ingreso, la paciente se encontró en buenas condiciones generales y en la exploración física se palpó adenomegalia cervical derecha de $4 \mathrm{~cm}$, de eje mayor. Se realizó biometría hemática que reportó leucocitos $8,4 \mathrm{miles} / \mathrm{mm}^{3}$, hemoglobina de $11,7 \mathrm{~g} / \mathrm{dl}$ y plaquetas $295 \mathrm{miles} / \mathrm{mm}^{3}$. Se realizó PET-CT que informó aumento anormal de la actividad del radiotrazador en los ganglios 
cervicales derechos (SUVmax 29,6) e izquierdos (SUVmax 7,5), retropectorales (SUVmax 23,2), mediastinales (SUVmax 4,19), iliacos (SUVmax 4,6), inguinales (SUVmax 6,8) y en ambas suprarrenales (SUVmax 28,7). En la revisión de los nuevos cortes del bloque de parafina del ganglio linfático axilar se observó pérdida de su arquitectura normal por expansión del espacio interfolicular y medular por células plasmáticas de aspecto maduro (Figura 1). Se realizó estudio de inmunohistoquímica el cual resultó positivo

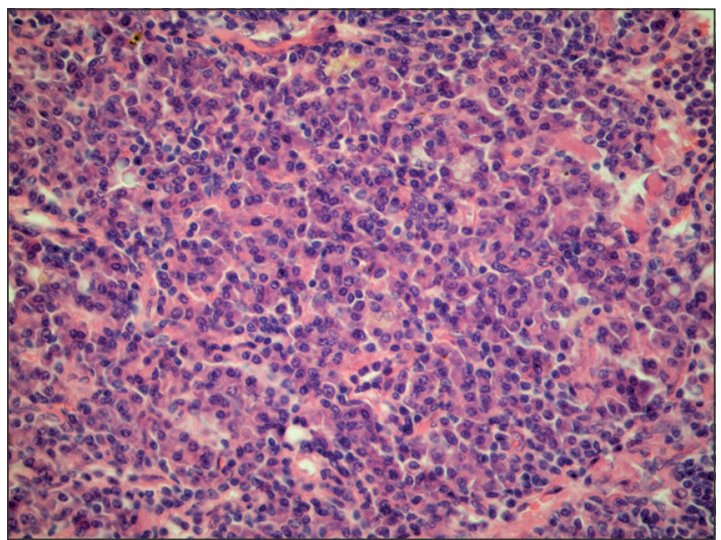

Figura 1. Biopsia de ganglio linfático que muestra mantos de células plasmáticas maduras que ocupan el espacio interfolicular (Hematoxilina-eosina, 400X).

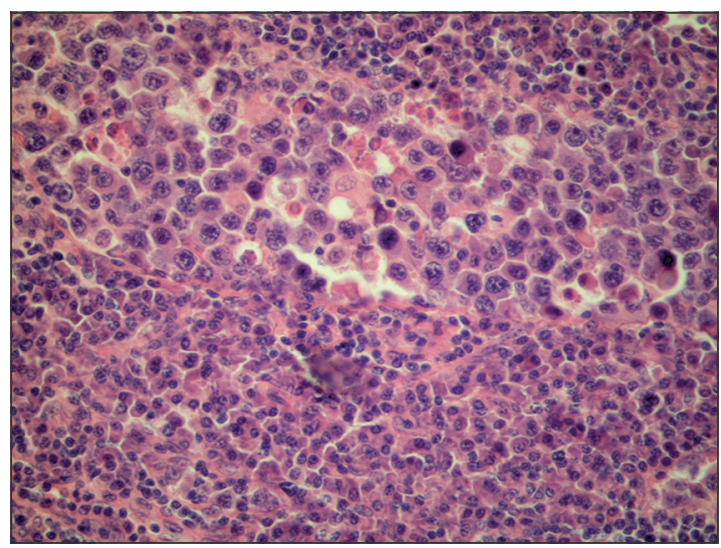

Figura 2. Grupos de células neoplásicas de aspecto plasmablástico rodeadas por células plasmáticas maduras. En estas áreas la atipia citológica es marcada con ocasionales figuras de mitosis (Hematoxilina-eosina, 400X). en las células plasmáticas para CD 138, kappa y lambda. De manera focal se identificaron áreas sólidas de bordes mal definidos, constituidas por células de núcleos grandes, nucléolo prominente y citoplasma amplio basófilo, que les confería un aspecto plasmablástico. En estas áreas la atipia citológica fue marcada con ocasionales figuras de mitosis (Figura 2). Se realizaron reacciones de inmunohistoquímica utilizando anticuerpos contra: CD 3 (Policlonal; 1:200 Dako), CD 20 (L26; 1:400 Dako), CD 38 (AT13/5; 1:25 DBS), CD 138 (MI15; 1:75 Dako), MUM-1 (MUM1p; 1:200 Dako), Kappa (Policlonal; 1:20.000 Dako), Lambda (Policlonal; 1:20.000 Dako), Ki-67 (EP5; 1:50 DBS) y HVSK/VHH8 (13B10; 1:20 Cell Marque). Las células neoplásicas expresaron CD 38, MUM-1, cadenas ligeras lambda y LANA-1 del HVSK/VHH8 (Figura 3). Se realizó EBER que resultó negativo, lo que corroboró el diagnóstico de LCGBEC. La prueba de ELISA para VIH y el perfil viral de hepatitis resultaron negativos. Además, se realizó aspirado y biopsia de médula ósea, ambas se informaron sin infiltración. Se estadificó en etapa III (Ann Arbor) e inició tratamiento con R-CHOP (rituximab $375 \mathrm{mg} / \mathrm{m}^{2}$, ciclofosfamida $750 \mathrm{mg} / \mathrm{m}^{2}$, clorhidrato de doxorrubicina $50 \mathrm{mg} /$ $\mathrm{m}^{2}$, vincristina $1,4 \mathrm{mg} / \mathrm{m}^{2}$ y prednisona $100 \mathrm{mg} /$ día/5 días) completando 8 ciclos. Actualmente, la paciente se encuentra en remisión, sin evidencia de enfermedad, después de 18 meses de seguimiento.

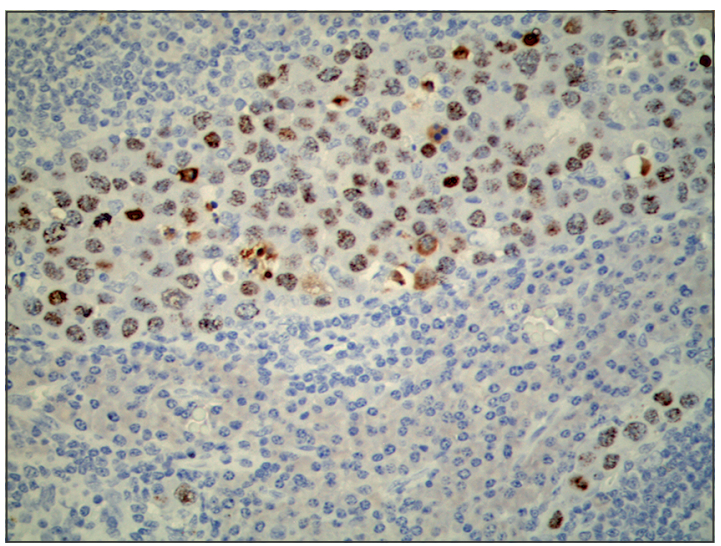

Figura 3. Expresión de LANA-1 del HVSKNHH8 en las células neoplásicas. Las células plasmáticas maduras son negativas (Técnica de inmunohistoquímica, 400X). 


\section{Discusión}

Desde la identificación del HVSK/VHH8 como agente causal del sarcoma de Kaposi (SK) en 1994, se ha asociado a diversos trastornos linfoproliferativos con características clínico-patológicas específicas, que incluyen diferentes inmunofenotipos y coinfección con el virus de Epstein-Barr (VEB) como: enfermedad de Castleman multicéntrica (ECM), linfoma primario de serosas y su variante sólida, proliferaciones plasmablásticas originadas en ECM, desorden linfoproliferativo germinotrópico; así como LCGBEC asociado a HVSK/ $\mathrm{HHV}^{2}$. La EC se caracteriza por linfadenopatía, fiebre, hipergammaglobulinemia, frecuentemente los pacientes muestran anormalidades inmunológicas y desarrollan tumores secundarios ${ }^{6}$. Aun cuando la mayoría de las lesiones son de origen policlonal y se consideran reactivas, se ha informado que bajo ciertas circunstancias los pacientes con EC del tipo difuso de células plasmáticas pueden progresar hacia diferentes tipos de linfomas no Hodgkin de células B como: linfoma del manto ${ }^{7}$, linfoma folicular ${ }^{8}$, LCGB $^{1,9,10}$, linfoma plasmablástico, linfoma primario de serosas y su variante sólida; así como linfoma de Hodgkin (LH) ${ }^{11-16}$. El HVSK/VHH8 codifica más de diez homólogos de genes celulares que regulan señales de proliferación celular y antiapoptóticas, que pudieran estar implicados en el desarrollo y progresión de estas neoplasias ${ }^{17-18}$.

El LCGBEC ocurre frecuentemente en pacientes $\mathrm{VIH}+$ que han desarrollado ECM asociada a HVSK/VHH8; sin embargo, existen casos informados en pacientes VIH- que han desarrollado ECM, usualmente en regiones geográficas donde el HVSK/VHH8 es endémico ${ }^{3,4}$. Erkurt et al. informaron el caso de una mujer de 66 años de edad VIH- con diagnóstico de ECM de tipo hialino vascular que desarrolló LCGB. La paciente recibió radioterapia en la región cervical con una dosis total de $4.000 \mathrm{cGy}$, seguida de seis ciclos de quimioterapia a base de $\mathrm{CHOP}$, con remisión completa por un período de seguimiento de 2 $a_{n}{ }^{19}{ }^{19}$. Larroche et al. publicaron una serie de ocho pacientes VIH- con EC tanto multicéntrica como localizada, asociados a linfomas de células B, que incluían: linfoma del manto, LCGB, linfoma linfoplasmacítico y $\mathrm{LH}^{16}$. El caso que informamos comparte características similares; es una paciente VIH- con diagnóstico de LCGBEC y excelente respuesta al tratamiento con $\mathrm{R}-\mathrm{CHOP}$, con un período libre de enfermedad de 18 meses.

Característicamente, estos linfomas involucran los ganglios linfáticos y el bazo, pero puede diseminarse a otros órganos, manifestándose en algunos pacientes como un cuadro leucémi$\mathrm{Co}^{9,10}$. Morfológicamente, los folículos linfoides muestran grados variables de involución y hialinización de los centros germinales con una zona del manto prominente que puede infiltrar y obliterar los centros germinales. En esta zona y en el espacio interfolicular se pueden observar un número variable de células grandes de citoplasma basófilo amplio y un núcleo grande vesicular localizado excéntricamente, que contiene uno o más nucléolos prominentes lo que les confiere un aspecto plasmablástico, alternando con numerosas células plasmáticas maduras. Conforme la enfermedad progresa, los plasmablastos forman grupos microscópicos confluentes, también llamados "microlinfomas", tanto dentro como fuera de los centros germinales ${ }^{9}$. En el estudio histopatológico del presente caso se identificó claramente la coexistencia de EC del tipo difuso de células plasmáticas y LCGB de aspecto plasmablástico. El origen de un franco linfoma es por medio de la expansión y confluencia de estos mantos que afectan la arquitectura normal de los ganglios linfáticos y/o bazo; así como hígado, pulmón, tracto gastrointestinal y, en algunos casos, sangre periférica ${ }^{9,10}$. El perfil de expresión característico de las células neoplásicas es IgM+, IL-6 viral+, MUM-1+, CD 38-/+, CD 138-, CD 79a-, CD 20-/+, LANA-1+ y EBER- ${ }^{5}$.

El diagnóstico diferencial incluye el trastorno linfoproliferativo germinotrópico $\mathrm{VHH} 8+$ / $\mathrm{VEB}+$. Estos pacientes generalmente son adultos inmunocompetentes VIH- y típicamente no se observan características morfológicas de la EC. Los plasmablastos observados en esta entidad son positivos para CD 38, CD 138, IRF4/MUM-1; así como para VHH-8 y VEB, y negativos para marcadores pan-B, CD 10, Bcl-2 y Bcl- $6^{20}$.

El linfoma folicular puede confundirse con un trastorno linfoproliferativo asociado a $\mathrm{VHH}$ 8 debido a la presencia de centroblastos en los folículos; sin embargo, las células neoplásicas del linfoma folicular son positivas para CD 20, CD $10, \mathrm{Bcl}-2$ y Bcl-6 y negativas tanto para $\mathrm{VHH}-8$ y $\operatorname{VEB}^{2,21-23}$.

Los casos de linfomas no Hodgkin asociados a EC comparten características comunes con lin- 
fomas observados en varios tipos de deficiencias inmunes: son linfomas de células B de grado alto, se diagnostican en estadios III o IV, tienen afección extraganglionar y curso clínico agresivo con supervivencia corta ${ }^{16}$.

Los linfomas originados en el contexto de EC deben ser tratados utilizando esquemas de quimioterapia estándar. En particular, los pacientes con LCGBEC muestran buena respuesta al esquema convencional de CHOP o R-CHOP, en algunos casos obteniendo remisión completa posterior al tratamiento ${ }^{15,16}$.

En conclusión, la EC es una entidad heterogénea con una fisiopatología compleja, que se ha asociado con diferentes tipos de linfomas, particularmente cuando es multicéntrica y se asocia a VIH. Por otro lado, la coexistencia de EC y linfomas en pacientes VIH- es rara y poco clara, con pocos casos informados; sin embargo, estos pacientes en general tienen un mejor pronóstico que los pacientes $\mathrm{VIH}+$, posiblemente como reflejo de una mejor función inmune. Es importante identificar las características morfológicas e inmunohistoquímicas de los linfomas originados en la EC, con la finalidad de establecer el diagnóstico correcto y proporcionar el tratamiento adecuado.

\section{Referencias}

1. Ogawa Y, Watanabe D, Hirota K, Ikuma M, Yajima $\mathrm{K}$, Kasai D, et al. Rapid multiorgan failure due to large B-cell lymphoma arising in human herpesvirus-8-associated multicentric Castleman's disease in a patient with human immunodeficiency virus infection. Intern Med 2014; 53 (24): 2805-9.

2. Courville EL, Sohani AR, Hasserjian RP, Zukerberg LR, Harris NL, Ferry JA. Diverse clinicopathologic features in human herpesvirus 8-associated lymphomas lead to diagnostic problems. Am J Clin Pathol 2014 (6); 142: 816-29.

3. Boshoff C, Weiss RA. Epidemiology and pathogenesis of Kaposi's sarcoma-associated herpesvirus. Philos Trans R Soc Lond B Biol Sci 2001; 356 (1408): 517-34.

4. Venizelos I, Tamiolakis D, Simopoulos C, Nikolaidou S, Barbagadaki S, Lambropoulou M, et al. Diffuse large B-cell lymphoma arising from a multicentric mixed variant of Castleman's disease. Indian J Cancer 2004; 41 (3): 135-7.

5. Swerdlow SH, Campo E, Harris NL, Jaffe ES, Pileri SA, Stein $\mathrm{H}$, et al. (Eds). WHO Classification of Tumours of Haematopoietic and Lymphoid Tissues. IARC; Lyon 2008.

6. Sobrevilla-Calvo P de J, Avilés-Salas A, Cortés-Padilla DE, Rivas-Vera S. Características clinicopatológicas de la enfermedad de Castleman. Experiencia en el Instituto Nacional de Cancerología. Cir Cir 2009; 77 (3): 187-92.

7. Yoshida T, Sakai A, Imagawa J, Noda M, Takada S, Yoshino T, et al. Mantle cell lymphoma superimposed on multicentric Castleman's disease. J Clin Exp Hematop 2011; 51 (2): 147-50.

8. Koreishi AF, Saenz AJ, Arcila ME, Hedvat C, Fleming S, Teruya-Feldstein J. Synchronous follicular lymphoma, Kaposi sarcoma, and Castleman's disease in a HIV-negative patient with EBV and HHV-8 coinfection. Int J of Surg Pathol 2011; 19 (5): 685-91.

9. Dupin N, Diss TL, Kellam P, Tulliez M, Du MQ, Sicard D, et al. HHV-8 is associated with a plasmablastic variant of Castleman disease that is linked to HHV-8 positive plamablastic lymphoma. Blood 2000; 95 (4): 1406-12.

10. Oksenhendler E, Boulanger E, Galicier L, Du MQ, Dupin N, Diss TC, et al. High incidence ok Kaposi sarcoma-associated herpesvirus-related non-Hodgkin lymphoma in patients with HIV infection and multicentric Castleman disease. Blood 2002; 99 (7): 2331-6.

11. Rao S, Ramesh A, Rajkumar A, Arcot R, Kuruvilla S. Persistent or recurrent Castleman's disease-look out for a lurking lymphoma! Indian J Med Paediatr Oncol 2011; 32 (3): 162-4.

12. Frank DK, Charney D, Kashani A. Plasma cell variant of Castleman's disease occurring concurrently with Hodgkin's disease in the neck. Head Neck 2001; 23 (2): 166-9.

13. Cesarman E, Knowles DM. Kaposi's sarcoma-associated herpesvirus: a lymphotropic human herpesvirus associated with Kaposi's sarcoma, primary effusion lymphoma, and multicentric Castleman's disease. Semin Diagn Pathol 1997; 14 (1): 54-66.

14. Scott MJ, Alhariri BC, Farghaly H, Pérez R, Arnold FW. Castleman's disease and primary effusion lymphoma in a HIV-positive patient. Int J STD AIDS 2014; 25 (6): 455-7.

15. Mohtaram A, Afif M, Sghiri T, Rami A, Latib R, Kettani F, et al. Coexistence of Hodgkin's lymphoma and Castleman's disease: a case report with successful response to chemotherapy and radiotherapy. Case Rep Oncol Med 2013; 2013: 487205. doi:10.1155/2013/487205.

16. Larroche C, Cacoub P, Soulier J, Oksenhendler E, Clauvel JP, Piette JC, et al. Castleman's disease and lymphoma: report of eight cases in HIV-negative patients and literature review. Am J Hematol 2002; 69 (2): 119-26. 
17. Arvanitakis L, Geras-Raaka E, Varma A, Gershengorn MC, Cesarman E. Human herpesvirus KSHV encodes a constitutively active G-protein-coupled receptor linked to cell proliferation. Nature 1997; 385 (6614): 347-50.

18. Järvilouna A, Koopal S, Räsänen S, Mäkelä TP, Ojala PM. KSHV viral cyclin binds to p27KIP1 in primary effusion lymphomas. Blood 2004; 104 (10): 3349-54.

19. Erkurt MA, Aydogdu I, Kuku I, Kaya E, Mizrak B, Basaran Y. A multicentric, hyaline vascular variant of Castleman's disease associated with B cell lymphoma: a case report. Cases J 2009; 2: 8183. doi:10.4076/17571626-2-8183.

20. Du MQ, Diss TC, Liu H, Ye H, Hamoudi RA, Cabecadas J, et al. KSHV-and EBV-associated germinotropic lymphoproliferative disorder. Blood 2002; 100 (9): 3415-8.
21. D'Antonio A, Boscaino A, Addesso M, Piris MA, Nappi O. KSHV- and EBV-associated germinotropic lymphoproliferative disorder: a rare lymphoproliferative disease of HIV patient with plasmablastic morphology, indolent course and favourable response to therapy. Leuk Lymphoma 2007; 48 (7): 1444-7.

22. Pan ZG, Zhang QY, Lu ZB, Quinto T, Rozenvald IB, Liu LT, et al. Extracavitary KSHV-associated large B-cell lymphoma: a distinct entity or a subtype of primary effusion lymphoma? Study of 9 cases and review of an additional 43 cases. Am J Surg Pathol 2012; 36 (8): 112940.

23. Montes-Moreno S, Montalbán C, Piris MA. Large B-cell lymphomas with plasmablastic differentiation: a biological and therapeutic challenge. Leuk Lymphoma 2012; 53 (2): 185-94. 